\title{
Diagnósticos de enfermagem para o paciente com necrólise epidérmica tóxica: estudo de caso
}

Nursing diagnoses for the patient with toxic epidermal necrolysis: a case study

Diagnósticos de enfermería para el paciente con necrólisis epidérmica tóxica: estudio de caso

\author{
Taize Muritiba Carneiro', Iranete Almeida Sousa Silva" \\ ' Universidade Federal da Bahia, Complexo Hospitalar Universitário Professor Edgard Santos. Salvador-BA, Brasil. \\ "Universidade Federal da Bahia, Complexo Hospitalar Professor Edgard Santos. \\ Faculdade Adventista da Bahia, Curso de Enfermagem. Salvador-BA, Brasil.
}

Submissão 03-01-2011 Aprovação: 24-01-2012

\section{RESUMO}

Trata-se de um estudo de caso clínico, retrospectivo, de um paciente acometido por necrólise epidérmica tóxica, internado em uma unidade de terapia intensiva de uma organização hospitalar pública, com o objetivo de apreender, a partir dos julgamentos clínicos das (os) enfermeiras (os), os diagnósticos de enfermagem. Foram evidenciados treze diagnósticos de enfermagem, assim como a necessidade de aprimoramento teórico destas profissionais sobre a Sistematização da Assistência de Enfermagem e o sentido de valor que esta prática pode acrescentar à profissão, na condução da assistência individualizada aos pacientes sob seus cuidados.

Descritores: Enfermagem; Necrólise epidérmica tóxica; Diagnósticos de enfermagem; Unidade de Terapia Intensiva.

ABSTRACT
This is a retrospective case study of a patient affected by toxic epidermal necrolysis in the intensive care unit of a public hospital, with the goal to apprehend, starting from the clinical judgments of the nurses, theirs nursing diagnoses. Thirteen nursing diagnoses were evidenced and, also, it was evidenced the necessity of the theoretical improvement of those professionals about the Systematization of Nursing Care, and on the sense of value that this practice may add to nursing in the pursuit of individualized assistance to the patients under their care.

Key words: Nursing; Epidermal necrolysis, toxic; Nursing diagnoses; Intensive Care Unit.

\section{RESUMEN}

Es un estudio de caso clínico, retrospectivo, de un paciente afectado por necrólisis epidérmica tóxica, internado en una unidad de terapia intensiva de una organización hospitalaria pública, con el objetivo de aprehender, a partir de los juicios clínicos de las(los) enfermeras(os), los diagnósticos de enfermería. Fueran evidenciados trece diagnósticos de enfermería, así como la necesidad de actualización teórico-práctica de estas profesionales, sobre la Sistematización de la Asistencia de Enfermería y el sentido de valor que esta práctica puede añadir a la Enfermería, en la conducción de la asistencia individualizada a los pacientes bajo sus cuidados.

Palabras clave: Enfermería; Necrólisis epidérmica tóxica; Diagnóstico de enfermería; Unidad de Terapia Intensiva. 


\section{INTRODUÇÃO}

O termo Necrólise Epidérmica Tóxica (NET), introduzido por Lyell no ano de 1956, também descrita como Síndrome de Lyell, caracteriza-se por um extenso descolamento da epiderme, secundário à necrose, e constitui uma reação adversa e rara a drogas ${ }^{(1)}$.

A NET possui como características iniciais sintomas inespecíficos como dor de garganta, febre e queimação ocular. Essas manifestações geralmente aparecem um a três dias antes do acometimento cutâneo ${ }^{(1)}$. As lesões cutâneas surgem na face e parte superior do tronco, associadas a queixas de queimação ou dor. Tais lesões, geralmente, são caracterizadas como máculas eritematosas, de contornos mal definidos, com um centro purpúreo, e evoluem para região anterior e dorso do tórax. O referido quadro pode evoluir em horas ou, muito raramente, no prazo de uma semana, para a extensão completa do corpo.

Na Europa e Estados Unidos da América (EUA), a NET ocorre em aproximadamente duas a três pessoas por milhão de habitantes ao ano. Acometem todas as idades, raças e sexo. A tendência de aparição, nos EUA, é na primavera e inverno. Dados brasileiros, em relação à sua prevalência são escas$\operatorname{sos}^{(2)}$. Essa síndrome é fatal em 30\% dos casos acometidos.

O aspecto clínico da NET tem semelhança à Síndrome de Stevens-Johnson, porém, nesta, o descolamento epidérmico é limitado a $10 \%$ da superfície corpórea. O diagnóstico deverá ser confirmado através de biópsia cutânea. Com a evolução da doença, o paciente apresenta um aspecto de grande queimado, com desequilíbrio hidroeletrolítico e perda acentuada de proteínas, podendo apresentar infecção concomitante e sepse.

As drogas que mais comumente podem causar a NET são as sulfas, o fenobarbital, a carbamazepina, a dipirona, o piroxicam, a fenilbutazona, as aminopenicilinas e o alopurinol ${ }^{(1)}$. O tratamento é semelhante ao dispensado ao paciente grande queimado. Este deverá receber cuidados em unidade de terapia intensiva ou unidade de queimados tais como: precauções para isolamento protetor, ambiente aquecido, prevenção de trauma cutâneo, suspensão de medicamentos não essenciais à vida, reposição de fluidos via parenteral na impossibilidade de reposição por via oral, aplicação de antissépticos líquidos como Nitrato de prata a $0,5 \%$ ou Clorhexidina a 0,05 , além de proteção de mucosa gástrica. A introdução de antibióticos está indicada em sinais de infecção ou aumento de bactérias cultivadas. Há relato de casos com o uso de imunoglobulina endovenosa com excelentes respostas ${ }^{(1)}$.

Face à gravidade da NET, à dificuldade das autoras na implementação da Sistematização da Assistência de Enfermagem (SAE) a um paciente com essa enfermidade e à lacuna na literatura de enfermagem sobre este assunto, tornou-se relevante a realização deste estudo, cujo enfoque é uma etapa do processo de enfermagem (PE) - o diagnóstico de enfermagem (DE).

O PE é um caminho metodológico, sistematizado e dinâmico para a assistência de enfermagem ao indivíduo, família ou comunidade. O mesmo possibilita o cuidado individualizado, garante a autonomia da (o) enfermeira (o) na tomada de decisão no processo de cuidar, assim como conduz as avaliações e suas consequências, o que pode promover maior cientificidade à Enfermagem. Partindo-se dessa compreensão, o PE deve ser operacionalizado em etapas inter-relacionadas, a partir da identificação de necessidades afetadas do paciente, da elaboração de diagnóstico, do planejamento das intervenções, sua implementação e avaliação.

No Brasil, na segunda metade dos anos 60, Wanda de Aguiar Horta, com base em sua teoria, denominada Teoria das Necessidades Humanas Básicas, apresentou às (os) enfermeiras (os) uma assistência de enfermagem sistematizada( ${ }^{(4)}$. Horta apresentou um modelo de PE composto de seis fases: histórico de enfermagem, diagnóstico de enfermagem, plano assistencial, plano de cuidados ou prescrição de enfermagem, evolução e prognóstico de enfermagem ${ }^{(5)}$. Este modelo continua sendo largamente utilizado atualmente, porém com outras denominações para algumas fases.

Ao longo do tempo, vários estudiosos têm apontado tanto as vantagens dos $D E$, quanto as dificuldades para a sua implementação $0^{(6)}$. Neste estudo, optou-se por essa fase do PE por verificar algumas dificuldades das (os) enfermeiras (os) da unidade quanto à realização e aceitação dessa ferramenta de trabalho em enfermagem.

Frente à singularidade da patologia e ausência de trabalhos na literatura de enfermagem brasileira sobre a NET, consideramos importante a elaboração deste estudo de caso clínico. Assim, esta pesquisa objetivou apreender os diagnósticos de enfermagem identificados em paciente acometido por necrólise epidérmica tóxica, internado em uma unidade de terapia intensiva (UTI) de uma organização pública de saúde.

\section{MÉTODO}

Trata-se de um estudo descritivo, retrospectivo, com abordagem qualitativa, do tipo estudo de caso clínico, o qual é bastante utilizado em enfermagem, principalmente com os alunos de graduação, por ser considerado um modelo de estudo que permite uma investigação minuciosa de um determinado caso, de um paciente como esse, preservando todas as características da vida real.

No estudo descritivo o pesquisador pretende descrever os fatos e os fenômenos de uma determinada realidade com exatidão e sem manipulação dos dados ${ }^{(7)}$. O estudo de caso clínico fundamenta as ações de enfermagem; proporciona uma assistência individualizada, através da qual o paciente é visto como um ser único e não apenas como um conjunto de sinais e sintomas ${ }^{(8)}$.

A coleta de dados foi realizada no período de 01 a 02 de abril de 2009, após aprovação do Comitê de Ética em Pesquisa da Universidade Federal da Bahia, Parecer n. 040/2008, e da UTI Geral Adulto de um Complexo Hospitalar Universitário público da cidade de Salvador-Ba. O estudo foi autorizado pelo familiar responsável pelo paciente, mediante assinatura do Termo de Consentimento pré-estabelecido, respeitando os princípios da Resolução 196/96 do Conselho Nacional de Saúde ${ }^{(9)}$. Os dados foram coletados a partir de registros de enfermagem contidos no prontuário do paciente estudado. 


\section{RELATO DE CASO}

Homem, 32 anos, solteiro, negro, natural do Estado da Bahia, trabalhador do campo, com diagnóstico colaborativo de NET. Sem relato de internações prévias. Admitido na UTI-G em 03/04/2008, proveniente do ambulatório de dermatologia, onde foi avaliado e evidenciado lesões bolhosas difusas por todo o corpo, associadas a descolamento de epiderme em face, membros superiores, inferiores e tórax. Nesse serviço, informou ter apresentado febre, associada a cefaléia e hiperemia ocular sete dias antes, quando fez uso de quatro comprimidos de diclofenaco, um comprimido a cada seis horas; e paracetamol, este sem registro de dosagem e frequência. Houve dificuldade para coleta de outros dados, como história familiar de doenças, uso de vacinas, hábitos de vida, entre outros, devido ao quadro clínico do paciente e ausência de familiar e/ou acompanhante durante a maior parte da hospitalização do mesmo.

No momento da admissão na UTI-G, apresentava estado geral comprometido, descorado, desidratado, acianótico, subfebril. Consciente, orientado, agitado, verbalizando com dificuldade, pupilas isocóricas e fotorreagentes, fácies de dor, lesões bolhosas e descolamento de epiderme em face e pavilhão auditivo, com couro cabeludo íntegro. Taquipneico, em respiração espontânea em ar ambiente, expansibilidade e simetria pulmonar preservadas, saturação venosa de $\mathrm{O}_{2}$ de $90 \%$, ausculta pulmonar com murmúrio vesicular presente, sem ruídos adventícios. Sem condições de mensurar pressão arterial não invasiva, por impossibilidade de colocação de manguito em membros, taquicárdico em ritmo sinusal, ausculta cardíaca: BRNF em 2T sem sopro, tórax apresentando lesões bolhosas com descolamento de epiderme. Apresentava abdome simétrico, com ruídos hidroaéreos presentes e lesões bolhosas com descolamento de epiderme. Com secreção purulenta em meato uretral e diurese concentrada. Possuía lesões bolhosas com descolamento de epiderme em membros superiores e inferiores. Leucograma 7100, Ht 43,7\%, $\mathrm{Hb} 14,9 \mathrm{mg} / \mathrm{dl}$, Plaquetas 270.000, Glicemia sérica $199 \mathrm{mg}$ / $\mathrm{dl}, \mathrm{K}+3,9$, Uréia $47 \mathrm{mg} / \mathrm{dl}$, Creatinina $0,9 \mathrm{mg} / \mathrm{dl}$. Gasometria arterial: $\mathrm{pH} 7,34, \mathrm{pCO}_{2} 45,6, \mathrm{pO}_{2} 222, \mathrm{BE}-1,8, \mathrm{HCO} 324,1$, Saturação arterialO $299,7 \%$.

\section{Análise dos dados e diagnósticos de enfermagem identificados}

Na referida UTI-G, a SAE foi implementada em todas as suas etapas, desde o ano 2006, como um projeto piloto para a reestruturação em todo o Complexo Hospitalar. Na rotina de trabalho da unidade está preconizado que os dados clínicos do paciente devem ser coletados durante a admissão, por meio da aplicação do histórico de enfermagem (HE). Este subsidia o julgamento clínico da (o) enfermeira (o) para a elaboração dos DE e, posteriormente, a prescrição de cuidados.

Tendo em vista os DE como um processo dinâmico e contínuo, considera-se importante atentar para a evolução diária e anotações da equipe de enfermagem sobre o paciente, para subsidiar atualizações que se fizerem necessárias destes DE e, posteriormente, realizar a prescrição diária de cuidados de enfermagem.
Como integrante do PE, o DE é a segunda etapa e, para sua realização, a (o) enfermeira (o) deverá coletar os dados sobre a saúde do paciente, sua família ou comunidade, analisá-los e interpretá-los criteriosamente. Para tal, essa (e) profissional precisa ter a capacidade de análise, julgamento, síntese e percepção ${ }^{(4)}$.

Neste estudo foram identificados treze diagnósticos de enfermagem elaborados pelas (os) enfermeiras (os) plantonistas, durante os dez dias de internação do paciente na UTI-G, os quais serão discutidos a seguir.

- Nutrição desequilibrada: menos do que as necessidades corporais, foi identificado em 09/04/08, relacionado à incapacidade de ingerir alimentos e perda protéica. A administração de alimentação, de forma precoce, via sonda nasoenteral ou parenteral, deverá ser instituída devido ao elevado gasto protéico-calórico ${ }^{(8)}$. Observamos que esse diagnóstico poderia ter sido identificado mais precocemente pelas (os) enfermeiras (os), já que o paciente não apresentava condições para alimentação oral, possuía perda líquida em grande área corporal e protéica, além do fato que a discussão em equipe para a introdução de dieta poderia interferir no quadro clínico do paciente, por reduzir também o risco de translocação bacteriana.

- $\quad$ Risco de infecção, identificado em 04/04/08, relacionado à pele rompida por lesões bolhosas e descolamento da epiderme, procedimento invasivo, por passagem de cateter venoso central para administração de líquidos e medicamentos e instalação de prótese para uso de ventilação mecânica invasiva. Esse diagnóstico perdurou durante toda a fase de internação do paciente.

- $\quad$ Risco de volume de líquidos deficiente, identificado em 04/04/08, estava relacionado à grande e continua eliminação de serosidade, devido a lesões de derme e epiderme que atingia grande área corporal e permaneceu durante toda sua internação na UTI-G.

- Integridade da pele prejudicada, identificado em 04/04/08, relacionado ao descolamento de epiderme e lesões bolhosas no corpo. Esse diagnóstico não foi resolvido, devido à permanência das lesões mesmo após as várias intervenções das equipes que assistiam o paciente.

- Comunicação verbal prejudicada, identificado em 09/04/08, estava relacionado à impossibilidade de verbalizar, em decorrência do uso de prótese orotraqueal para ventilação mecânica invasiva e sedação contínua. Esse diagnóstico não foi resolvido, pois o paciente permaneceu em uso dessa prótese durante o período que ficou internado na unidade; também poderia ter sido identificado desde sua entrada na unidade, já que há registros que, nesse dia, o paciente não conseguia verbalizar, por desconforto respiratório e dor, nesse mesmo dia foi instalada ventilação mecânica invasiva.

- Dor aguda, identificado em 04/04/08, relacionado a lesões por todo o corpo. Esse diagnóstico foi mantido pelas 
(os) enfermeiras (os) durante todo o internamento, apesar do uso contínuo de analgesia sob bomba infusora. Salientamos que a unidade não trabalhava com nenhuma escala de dor e, como o paciente também permaneceu sob sedação por um período prolongado, esse diagnóstico poderia ter sido acompanhado de forma mais evidente.

- Padrão respiratório ineficaz, identificado em 04/04/08, estava relacionado à dor intensa, fadiga da musculatura respiratória e hiperventilação. Foi parcialmente resolvido em 05/04/08, após instituição de ventilação mecânica invasiva.

- Troca de gases prejudicada, identificado em 04/04/08, relacionada ao desequilíbrio ventilação-perfusão evidenciado, após análise de hemogasometria arterial. Não foi encontrado registro de resolução nos impressos.

- Processos familiares interrompidos, identificado em 04/04/08, relacionado à internação em UTI, onde não há possibilidade de permanência de acompanhante; as visitas são em horários pré-estabelecidos, o domicilio do paciente era em cidade bastante distante desse nosocômio, além de dificuldades particulares de familiares para a visitação. Esse diagnóstico permaneceu durante toda a hospitalização. Não encontramos registro de visita de familiares para esse paciente.

- Termorregulação ineficaz, identificado em 09/04/08, relacionado à perda da epiderme em grande área corporal. Além disso, o desequilíbrio termorregulatório pode ocorrer por febre alta ou hipotermia, até a ausência de infecção ou cicatrização completa das lesões ${ }^{(1)}$. Permaneceu durante toda a hospitalização.

- Mobilidade física prejudicada, identificado em 04/04/08, relacionado à dor aguda, perda da integridade da pele em grande parte do corpo. Esse diagnóstico permaneceu durante toda a internação do paciente nessa unidade, já que não houve melhora das lesões na pele.
- Eliminação urinária prejudicada, identificado em 04/04/08, relacionado à infecção no trato urinário. Não havia registro de resolução.

- Perfusão tissular ineficaz, identificado em 04/04/08, relacionado a transporte prejudicado de oxigênio, evidenciado em resultado de hemogasometria arterial, hipovolemia, devido à grande perda de líquidos por perda de grande área da epiderme.

\section{CONSIDERAÇÕES FINAIS}

O PE, caracterizado como uma aplicação sistemática do conhecimento a situações específicas da assistência de enfermagem aos pacientes, foi empregado neste estudo e possibilitou a identificação de treze DE, a partir de julgamentos clínicos realizados por enfermeiras (os) de uma UTI-G de adultos.

Ficou evidenciada a necessidade de conhecimento teórico desses profissionais sobre a SAE e o sentido de valor que esta prática pode dar aos profissionais na condução da assistência individualizada aos pacientes sobre seus cuidados, neste estudo, especificamente, os portadores de NET.

É importante considerar que a admissão de pacientes nesta UTI sem a devida coleta de dados e histórico de enfermagem por suas unidades de origem são fatores que podem desestimular as (os) profissionais enfermeiras (os) na elaboração dos $\mathrm{DE}$, haja vista a dificuldade de acessá-los diante de sua criticidade, assim como a seus familiares.

Entretanto, além do incentivo indispensável dos gestores de enfermagem do setor e da unidade hospitalar, considera-se mais importante ainda, a atitude dessas (es) profissionais em valorizar o PE, compreender a visibilidade e autonomia que esse pode conferir à profissão e consequentemente o engajamento pessoal no sentido de romper o paradigma da impossibilidade de execução do processo de forma plena.

Sem a pretensão de esgotar o tema, acredita-se que a identificação dos DE proposta neste estudo poderá direcionar a assistência de enfermagem a pacientes com NET e estimular novas pesquisas para aprofundamentos dessa temática.

\section{REFERÊNCIAS}

1. Criado PR et al. Reações cutâneas graves adversas a drogas - aspectos relevantes ao diagnóstico e ao tratamento Parte I - anafilaxia e reações anafilactóides, eritrodermias e o espectro clínico da síndrome de Stevens-Johnson \& necrólise epidérmica tóxica (Doença de Lyell). An Bras Dermatol 2004;79(4): 471-88.

2. Bulisani ACP et al. Síndrome de Stevens-Johnson e necrólise epidérmica tóxica em medicina intensiva. Rev Bras Ter Intensiva 2006;18(3):292-7.

3. Faeda A, Leon CGRMP. Assistência de enfermagem a um paciente portador de Diabetes Mellitus. Rev Bras Enferm 2006;59(6):818-21.
4. Tannure MC, Gonçalves AMP. SAE, Sistematização da assistência de enfermagem: guia prático. Rio de Janeiro: Guanabara Koogan; 2008.

5. Horta WA. Processo de Enfermagem. São Paulo: EPU; 1979.

6. Kruse MHL, Silva KS, Ribeiro RG, Fortes CV. Ordem como tarefa: a construção dos Diagnósticos de Enfermagem. Rev Bras Enferm 2008;61(2):262-6.

7. Triviños ANS. Introdução à pesquisa em ciências sociais: a pesquisa qualitativa em educação. São Paulo: Atlas; 2007. 
8. Galdeano LE, Rossi LA, Zago MMF. Roteiro instrucional para a elaboração de um estudo de caso clínico. Rev Latinoam Enferm 2003;11(3):371-5.
9. Conselho Nacional de Saúde (Brasil). Resolução 196/96. Diretrizes e normas regulamentadoras de pesquisas envolvendo seres humanos. Rio de Janeiro: Fiocruz, 1998. 